\title{
Determining both adhesion energy and residual stress by measuring the stiction shape of a microbeam
}

\author{
Yin Zhang $\cdot$ Ya-Pu Zhao
}

Received: 8 February 2014 / Accepted: 11 March 2014 / Published online: 30 March 2014

(C) Springer-Verlag Berlin Heidelberg 2014

\begin{abstract}
The competition between the adhesive force and the beam restoring force determines the stiction shape of a microbeam. The presence of residual stress changes the beam stiffness and thus leads to the change of the beam restoring force. This study presents a model of incorporating the residual stress effect for the beam stiction. The previous models of arc-shape and S-shape correspond to the zero residual stress case, which also prescribes the stiction shape. When the residual stress becomes large, arc-shape and S-shape significantly deviate from the actual stiction shape of a slender beam. With the assumed stiction shape of arc-shape and S-shape, suspension length is the only parameter needed to characterize the stiction shape and suspension length can also be used to uniquely determine the adhesion energy. However, there are infinite combinations of residual stress and adhesion energy which can result in the same suspension length. Besides suspension length, the beam rise above the substrate can also be used as a parameter to characterize the stiction shape. This study presents a method of using these two parameters to uniquely determine the residual stress and adhesion energy as an inverse problem. A computation technique of using the stiction shape symmetry to significantly reduce the computation is also demonstrated.
\end{abstract}

\section{Introduction}

Suspended micromechanical structures have been extensively used in varieties of microsensors and microactuators,

Y. Zhang $(\bowtie) \cdot$ Y.-P. Zhao

State Key Laboratory of Nonlinear Mechanics (LNM), Institute of Mechanics, Chinese Academy of Sciences, Beijing 100190, China

e-mail: zhangyin@1nm.imech.ac.cn for example, contact switch (Loh and Espinosa 2012). In general, it is desirable to make sensors and actuators that have a minimum gap distance and a large surface area (Yang 2006). Smaller gap distance requires smaller actuation voltage, less power consumption and less amount of energy stored in the system. If the stored energy is large, the discharge current densities during the contact of suspended structure and substrate can be so large to ablate the active element or damage the electrode, which is the so-called burn-out phenomenon (Loh and Espinosa 2012). A straightforward solution to burn-out is the smaller gap distance. However, such solution comes at the expense of favoring the stiction failure mode. Use of dimples or cavities ( $\mathrm{Li}$ et al. 2010; Yang 2006) to reduce contact area, or hydrophobic surface coating to reduce surface energy (Loh and Espinosa 2012; Maboudian and Howe 1997), or to operate the device in dry or vacuum environment to reduce capillary force (Wei and Zhao 2007; Wu et al. 2011), can not completely prevent stiction from occurring during the release process or in-use applications because of the presence of van der Waals (vdW) force (DelRio et al. 2005). Stiction is a major failure mechanism for the microelectromechanical systems (MEMS) structures and extensive studies have been done on this topic. Stiction is the competition result of the microstructure elastic energy and microstructure-substrate interfacial energy, i.e., the work of adhesion (de Boer and Michalske 1999; Knapp and de Boer 2002; Legtenberg et al. 1994; Maboudian and Howe 1997; Mastrangelo and Hsu 1993a, b; Zhang and Zhao 2011, 2012).

The presence of residual stress and its gradients in a microstructure, which in essence changes the stiffness and elastic energy of the microstructure (Zhang and Zhao 2006), is a common phenomenon. As a direct outgrowth of silicon-based microelectronics, the manufacturing technique of using successively patterned deposition of thin-film 
polysilicon and sacrificial oxide layers is applied to fabricate those MEMS structures (de Boer and Michalske 1999). Polysilicon is prone to have residual stress/gradients, which is strongly dependent on the deposition process and heating cycles, including doping or annealing (Yee et al. 2002). For a clamped-clamped microstructure (Legtenberg et al. 1994; Li et al. 2010; Maboudian and Howe 1997; Yee et al. 2002; Zhang and Zhao 2012), the axial thermoelastic stress due to the temperature variation also has the impact on the microstructure stiction (Yee et al. 2002). Even for a cantilever, when the stiction occurs with the S-shape configuration, which in essence is also a clamped-clamped configuration (de Boer and Michalske 1999; Mastrangelo and Hsu 1993a; Zhang and Zhao 2011), thermoelastic stress is also shown to have an influence on the microstructure stiction (Rogers et al. 2002). Residual stress can be the dominant factor in the microstructure deflection and stiction (Wong et al. 2007). Large residual stress makes the deflection of a plate more like a membrane one (Komaragiri et al. 2005; Wong et al. 2007). For example, Yang (2006) modeled the stiction of a microstructure with (large) residual stress as a membrane structure. The previous studies on the stiction of a mircostructure either assume the zero residual stress (de Boer and Michalske 1999; Legtenberg et al. 1994; Li et al. 2010; Mastrangelo and Hsu 1993a, b; Zhang and Zhao 2004, 2011, 2012), or the residual/thermoelastic stress is known as a measured quantity (Rogers et al. 2002; Wong et al. 2007; Yee et al. 2002), or a control parameter (Majidi et al. 2005). However, residual stress/gradients and thermoelastic stress in general are not known a priori. Thermoelastic stress is obtained by measuring the material coefficient of thermal expansion (CTE) and the temperature variation (Rogers et al. 2002; Yee et al. 2002). When the residual stress gradients are asymmetric along the microstructure thickness, which generates bending moment and thus deflection, the residual stress and its gradients can be characterized as a function of the microstructure dimensions (Zhang and Zhao 2006) or thermoelastic stress/temperature (Chen et al. 2002). When the residual stress gradients are symmetric along the microstructure thickness and no deflection occurs, bulge test is often used to extract the residual stress inside a microstructure (Vlassak and Nix 1992). By any standard, measuring the residual stress and its gradients or thermoelastic stress inside a microstructure is not a trivial thing. Extra experimental setup to monitor the temperature variation and heating devices are often needed in the thermoelastic stress measurement (Chen et al. 2002; Rogers et al. 2002). Multiple special specimens are needed (Vlassak and Nix 1992; Zhang and Zhao 2006); the extraction of residual stress and its gradients often involves complex numerical simulation (Chen et al. 2002; Zhang and Zhao 2006). In a bulge test, a pneumatic device is needed to exert a uniform pressure to deform the microstructure (Vlassak and Nix 1992). Furthermore, bulge test requires the microstructure in test to have a large deflection to extract residual stress, which involves complex nonlinear mechanics analysis (Komaragiri et al. 2005; Vlassak and Nix 1992; Wong et al. 2007). For the MEMS structure with a small gap distance, the requirement of large deflection can not be satisfied.

Besides the residual stress/gradients and thermoelastic stress, the nominal adhesion energy is another unknown material property in a stiction test. The nominal adhesion energy is found by minimizing the system total energy with respect to the suspension length (de Boer and Michalske 1999; Legtenberg et al. 1994; Maboudian and Howe 1997; Mastrangelo and Hsu 1993a; Yang 2004; Yee et al. 2002; Zhang and Zhao 2011, 2012). The total energy is the sum of the elastic energy and surface energy. The residual stress and thermoelastic stress generate axial force; the residual stress gradients generate bending moment. They all result in the change of the microstructure elastic energy. When the residual stress/gradients and thermoelastic stress are (assumed) zero or measured, the elastic energy can be calculated and the total energy minimization gives the relation of adhesion energy and detachment length. Such relation is given as follows for a cantilever beam with zero axial stress

$\gamma_{s}=C \frac{E_{1} H^{2} t^{3}}{S^{4}}$,

where $\gamma_{s}$ is the nominal adhesion energy, $E_{1}$ and $t$ are the beam Young's modulus and thickness, respectively. $H$ is the gap distance; $S$ is called the suspension length or the detachment length. $C$ is a constant, $C=3 / 8$ for the arc-shaped stiction (Maboudian and Howe 1997; Mastrangelo and Hsu 1993b) and $C=3 / 2$ for the S-shaped stiction (de Boer and Michalske 1999; de Boer et al. 1999; Yang 2004). Clearly, Eq. (1) presents an one-to-one relationship of adhesion energy and suspension length: once the suspension length is measured, the adhesion energy is uniquely determined. Similarly, if the nonzero residual axial stress/strain is measured, the adhesion energy can also be uniquely determined by the suspension length (Maboudian and Howe 1997; Yee et al. 2002). When the axial stress is unknown, as shown in this study, the one-to-one relationship can no longer hold: there are infinite combinations of the axial stress and adhesion energy which can result in the same suspension length. For any given $E_{1}, t$ and $H$, as indicated by Eq. (1) for the zero axial stress case, the suspension length $(S)$ is the only parameter needed to be measured in a stiction test.

Mathematically, the reason for no need to measure the out-of-plane deflection profile is that the deflection shape is assumed (Zhang and Zhao 2011, 2012). As shown in this study, the assumed deflection shapes such as arc-shape and S-shape, which are the zero axial load case, can not capture the stuck beam deflection shape when the axial stress is relatively large. On the other hand, the mature measurement 
Fig. 1 a Schematic diagram of a clamped-clamped beam under a concentrated transverse load $P$ and an axial load $T$. The coordinate system is also shown. $\mathbf{b}$ Arc-shape and S-shape

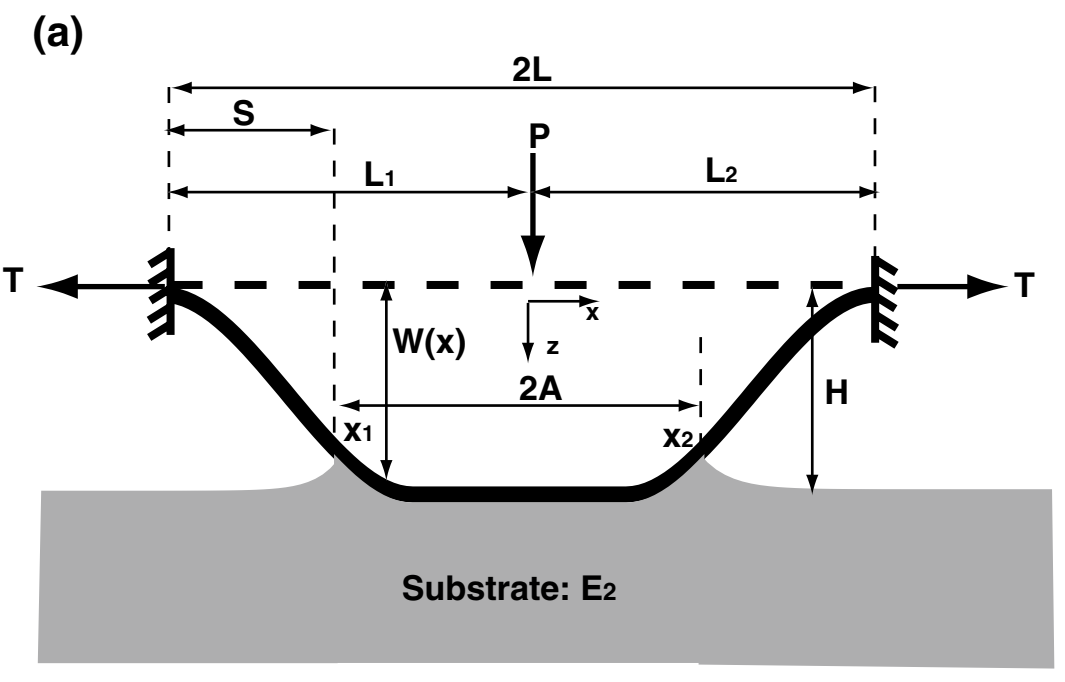

(b)

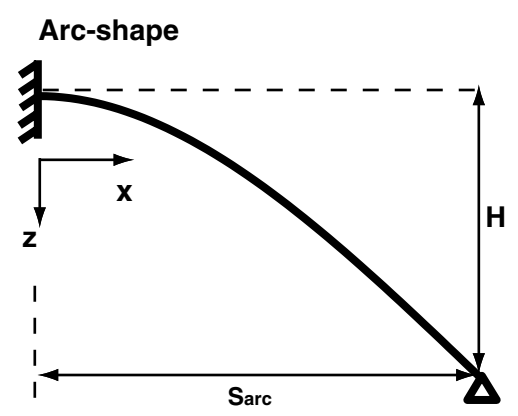

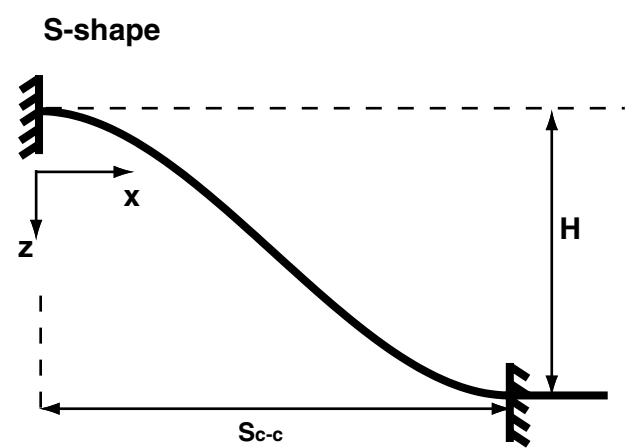

technique of Michelson interferometer is often used in a stiction test (de Boer and Michalske 1999; Jensen et al. 2001; Mastrangelo and Hsu 1993b) and the out-of-plane deflection of a stuck microstructure can be measured with the high accuracy of nanometer scale (Jensen et al. 2001). The deflection profile of a stuck microbeam is used to identify the structural nonidealties such as the compliance of support post (DelRio et al. 2006; Jensen et al. 2001). When the axial stress and adhesion energy are given, the suspension length is uniquely determined as a forward problem. However, in a stiction test, the axial stress and adhesion energy are the unknowns; the suspension length and deflection profile are the measured quantities (de Boer and Michalske 1999; Legtenberg et al. 1994; Maboudian and Howe 1997; Mastrangelo and Hsu 1993a, b; van Spengen et al. 2004). Therefore, in practice, the following inverse problem is encountered: how to use the suspension length and deflection profile to determine the axial stress and adhesion energy? This study presents the solution to this inverse problem. One obvious advantage of solving this inverse problem is that there is no need for extra experimental setup to measure residual stress or thermoelastic stress in a stiction test.

\section{Model development}

Figure 1a shows a clamped-clamped (C-C) beam under a concentrated load $P$ and an axial load $T$. The coordinate system is also shown in the figure, which coincide with the transverse load. The beam is with the length of $2 L$ and separated from substrate with the gap distance of $H$. When stiction occurs, some portion of the beam is in contact with the substrate. The beam displacement $W=W(x)$ is thus divided into three zones as shown in Fig. 1a: $W_{2}$ is the beam displacement in the contact zone; $W_{1}$ and $W_{3}$ are the displacements in the suspension zones (Weitsman 1970; Zhang and Murphy 2004). For brevity, the governing equations for the beam under an asymmetric load $P$ are given as the following

$$
\left\{\begin{array}{l}
E_{1} I \frac{d^{4} W_{1}}{d x^{4}}-T \frac{d^{2} W_{1}}{d x^{2}}=0, \quad-L_{1}<x<x_{1} \\
E_{1} I \frac{d^{4} W_{2}}{d x^{4}}-T \frac{d^{2} W_{2}}{d x^{2}}+k\left(W_{2}-H\right)=P \delta(x), \quad x_{1} \leq x \leq x_{2} \\
E_{1} I \frac{d^{4} W_{3}}{d x^{4}}-T \frac{d^{2} W_{3}}{d x^{2}}=0, \quad x_{2}<x<L_{2}
\end{array}\right.
$$

$E_{1}$ is the beam Young's modulus, $I$ is the area moment of inertia defined as $I=b t^{3} / 6$ (2b: the beam width and $t$ : the beam thickness). $\delta(x)$ is the Dirac delta function. $2 L$ is the 
beam length, $L_{1} / L_{2}$ is the distance from the left/right clamped end to the concentrated load and $2 L=L_{1}+L_{2} \cdot x_{1}$ and $x_{2}$ are the contact separation points, which are the unknowns to be determined. $x_{1}$ and $x_{2}$ are also the crack tip locations from the viewpoint of fracture mechanics (de Boer and Michalske 1999; de Boer et al. 1999; Knapp and de Boer 2002; Jones et al. 2003). $x_{2}-x_{1}=2 A$ is the beam attachment length and $S=L-A$ is the suspension length. $T$ is the beam axial load. As mentioned above, thermoelastic stress, residual stress and its symmetric gradients generate axial load; the asymmetric stress gradients generate bending moment (Zhang and Zhao 2006). Therefore, the above model only accounts the axial load effect of the residual stress and thermoelastic stress. However, as proved by Knapp and de Boer (2002), the bending moment only changes the initial equilibrium configuration and has no impact on the stiction behavior of a beam (in the small linear deformation range). In practice, there is no need to measure the initial equilibrium configuration or curvature due to the bending moment of asymmetric residual stress gradients (Knapp and de Boer 2002). The contact between the beam and substrate is modeled by the elastic foundation model and $k$ is the modulus of elastic foundation. $-k\left(W_{2}-H\right)$ is thus the contact pressure exerted by the substrate on the beam, which was also demonstrated to have impact on the equilibrium configuration of a stuck beam (Zhang and Zhao 2011, 2012). As shown in appendix, the contact pressure between beam and substrate is (implicitly) assumed zero in both the arc-shape and S-shape models. The contact pressure can also have the impact on the nominal adhesion energy. For example, Jones et al. (2003) observed that the nominal adhesion energy increases after several loading/unloading cycles. The explanation is that the contact pressure during the loading process induces the plastic deformation of the contacting surface asperities (Wu et al. 2011, 2013; Zhang and Zhao 2012), which increases the actual contact area and as a result of that $\gamma_{s}$ increases.

For a rectangular beam indenting a substrate which is modeled as an elastic half space, $k$ is given as follows (Biot 1937)

$k=0.71 E_{2}\left(\frac{E_{2} b^{4}}{E_{1} I}\right)^{1 / 3}$.

$E_{2}$ is the Young's modulus of substrate. The following nondimensionalization scheme is introduced (Weitsman 1970; Zhang and Murphy 2004; Zhang 2008)

$$
\begin{aligned}
& \xi=\beta x, \quad w_{i}=\beta W_{i}(i=1,2,3), \quad \xi_{1}=\beta x_{1}, \\
& \quad \xi_{2}=\beta x_{2}, \quad h=\beta H, \quad l_{1}=\beta L_{1}, \\
& l_{2}=\beta L_{2}, \quad l=\beta L, \quad \Delta=\frac{T}{E_{1} I \beta^{2}}, \quad F=\frac{P}{4 \beta^{2} E_{1} I}
\end{aligned}
$$

$\beta$ is defined as $\beta=\sqrt[4]{\frac{k}{4 E_{1} I}}$ (Weitsman 1970; Zhang and Murphy 2004), which has the unit of $\mathrm{m}^{-1}$. Physically, $\beta^{-1}$ is also the length used to evaluate the effect of beam bending on the contact (Castillo and Barber 1997). The dimensionless quantity $F$ indicates the ratio of external concentrated load to the beam transverse stiffness (Zhang and Zhao 2011, 2012); $\Delta$ indicates the (order of) ratio of axial load to the beam transverse stiffness.

Now Eq. (2) is nondimensionalized as the following

$\left\{\begin{array}{l}w_{1 \xi \xi \xi \xi}-\Delta w_{1 \xi \xi}=0, \quad-l_{1}<\xi<\xi_{1} \\ \frac{1}{4} w_{2 \xi \xi \xi \xi}-\Delta w_{2 \xi \xi}+w_{2}-h=F \delta(\xi), \quad \xi_{1} \leq \xi \leq \xi_{2}(5) \\ w_{3 \xi \xi \xi \xi}-\Delta w_{3 \xi \xi}=0, \quad \xi_{2}<\xi<l_{2} .\end{array}\right.$

Here ()$_{, \xi}=d / d \xi$ and $\Delta=T /\left(E_{1} I \beta^{2}\right)$ as given in Eq. (4). The solution forms to Eq. (5) are dependent on $\Delta$. For the zero axial load case of $\Delta=0$, the solution forms are given as follows (Weitsman 1970; Zhang and Murphy 2004)

$w=\left\{\begin{array}{c}w_{1}=A_{1} \xi^{3}+B_{1} \xi^{2}+C_{1} \xi+D_{1} \\ w_{2}=A_{2} \cosh \xi \sin \xi+B_{2} \cosh \xi \cos \xi+C_{2} \sinh \xi \sin \xi \\ +D_{2} \sinh \xi \cos \xi+\frac{F}{2}(\cosh \xi \sin |\xi|-\sinh |\xi| \cos \xi)+h \\ w_{3}=A_{3} \xi^{3}+B_{3} \xi^{2}+C_{3} \xi+D_{3}\end{array}\right.$

$A_{i}, B_{i}, C_{i}$ and $D_{i}(i=1,2$ and 3$)$ are twelve unknown constants. These twelve unknowns together with $\xi_{1}$ and $\xi_{2}$ are the fourteen unknowns in total to be solved.

For the tensile load case of $\Delta>0$, the solution forms are given as follows

$w=\left\{\begin{array}{c}w_{1}=A_{1} \cosh (\alpha \xi)+B_{1} \sinh (\alpha \xi)+C_{1} \xi+D_{1} \\ w_{2}=A_{2} \cosh \left(f_{1} \xi\right) \sin \left(f_{2} \xi\right)+B_{2} \cosh \left(f_{1} \xi\right) \cos \left(f_{2} \xi\right) \\ \quad+C_{2} \sinh \left(f_{1} \xi\right) \sin \left(f_{2} \xi\right)+D_{2} \sinh \left(f_{1} \xi\right) \cos \left(f_{2} \xi\right) \\ \quad+\frac{F}{2 f_{1} f_{2}}\left[\cosh \left(f_{1} \xi\right) \sin \left|f_{2} \xi\right|-\sinh \left|f_{2} \xi\right| \cos \left(f_{1} \xi\right)\right]+h \\ w_{3}=A_{3} \cosh (\alpha \xi)+B_{3} \sinh (\alpha \xi)+C_{3} \xi+D_{3}\end{array}\right.$

where $\alpha=\sqrt{\Delta}, \quad f_{1}=\sqrt{2} \cos \frac{\theta_{t}}{2}, \quad f_{2}=\sqrt{2} \sin \frac{\theta_{t}}{2} \quad$ and $\theta_{t}=\arctan \left(\sqrt{\frac{16-\Delta^{2}}{\Delta^{2}}}\right)$. For the compressive load case of $\Delta<0$, the solution forms are given as follows

$$
w=\left\{\begin{array}{c}
w_{1}=A_{1} \cos (\alpha \xi)+B_{1} \sin (\alpha \xi)+C_{1} \xi+D_{1} \\
w_{2}=A_{2} \cosh \left(f_{1} \xi\right) \sin \left(f_{2} \xi\right)+B_{2} \cosh \left(f_{1} \xi\right) \cos \left(f_{2} \xi\right) \\
\quad+C_{2} \sinh \left(f_{1} \xi\right) \sin \left(f_{2} \xi\right)+D_{2} \sinh \left(f_{1} \xi\right) \cos \left(f_{2} \xi\right) \\
\quad+\frac{F}{2 f_{1} f_{2}}\left[\cosh \left(f_{1} \xi\right) \sin \left|f_{2} \xi\right|-\sinh \left|f_{2} \xi\right| \cos \left(f_{1} \xi\right)\right]+h \\
w_{3}=A_{3} \cos (\alpha \xi)+B_{3} \sin (\alpha \xi)+C_{3} \xi+D_{3}
\end{array}\right.
$$

Now $\alpha=\sqrt{-\Delta}, \quad f_{1}=\sqrt{2} \cos \frac{\theta_{c}}{2}, \quad f_{2}=\sqrt{2} \sin \frac{\theta_{c}}{2} \quad$ and $\theta_{c}=\pi-\arctan \left(\sqrt{\frac{16-\Delta^{2}}{\Delta^{2}}}\right)$. When $\Delta=0, \theta_{t}=\theta_{c}=\pi / 2$ and $f_{1}=f_{2}=1$ for both the tensile and compressive load cases, $w_{2}$ solution forms recover those in Eq. (6) of the zero axial load case.

To solve the fourteen unknowns, fourteen equations are needed. At the contact separation points of $x_{1}$ and $x_{2}$, the following eight matching conditions must be satisfied 


$$
\begin{gathered}
w_{1}\left(\xi_{1}\right)=w_{2}\left(\xi_{1}\right), \quad \frac{d w_{1}}{d \xi}\left(\xi_{1}\right)=\frac{d w_{2}}{d \xi}\left(\xi_{1}\right), \\
\frac{d^{2} w_{1}}{d \xi^{2}}\left(\xi_{1}\right)=\frac{d^{2} w_{2}}{d \xi^{2}}\left(\xi_{1}\right), \quad \frac{d^{3} w_{1}}{d \xi^{3}}\left(\xi_{1}\right)=\frac{d^{3} w_{2}}{d \xi^{3}}\left(\xi_{1}\right), \\
w_{2}\left(\xi_{2}\right)=w_{3}\left(\xi_{2}\right), \quad \frac{d w_{2}}{d \xi}\left(\xi_{2}\right)=\frac{d w_{3}}{d \xi}\left(\xi_{2}\right), \\
\frac{d^{2} w_{2}}{d \xi^{2}}\left(\xi_{2}\right)=\frac{d^{2} w_{3}}{d \xi^{2}}\left(\xi_{2}\right), \quad \frac{d^{3} w_{2}}{d \xi^{3}}\left(\xi_{2}\right)=\frac{d^{3} w_{3}}{d \xi^{3}}\left(\xi_{2}\right) .
\end{gathered}
$$

The matching conditions can be obtained by applying the principle of virtual work. It is noticed that the above matching conditions are the same as those with zero axial load (Zhang and Murphy 2004; Zhang 2008). The same matching conditions are also obtained by Lin and Adams (1987) in the tensionless contact of a beam under an axial load, by Ghatak et al. $(2004,2005)$ in a film peeled from a substrate, and by Jiang et al. (2008) in the buckling of film on substrate. The matching conditions of Eq. (9) are also referred to as the transversality conditions (Kerr 1976; Liu 2010). The four boundary conditions of a $\mathrm{C}-\mathrm{C}$ beam are given as follows

$$
\begin{gathered}
w\left(-l_{1}\right)=0, \quad \frac{d w}{d \xi}\left(-l_{1}\right)=0, \\
w\left(l_{2}\right)=0, \quad \frac{d w}{d \xi}\left(l_{2}\right)=0 .
\end{gathered}
$$

Here we assume the rigid support post by setting the zero rotation angle of $d w / d \xi\left(-l_{1}\right)=0$. The rotation angle may not be zero due to the compliance of support post (de Boer and Michalske 1999; DelRio et al. 2006; Jensen et al. 2001).

Two constraint conditions are given as follows (Zhang and Zhao 2011, 2012)

$w_{2}\left(\xi_{1}\right)=h-\frac{\sqrt{\alpha_{r}}}{2}, \quad w_{2}\left(\xi_{2}\right)=h-\frac{\sqrt{\alpha_{r}}}{2}$.

where $\alpha_{r}$ is a dimensionless parameter defined as $\alpha_{r}=4 b \gamma_{s} /\left(E_{1} I \beta^{2}\right)$ (Zhang and Zhao 2011, 2012). Physically, $\sqrt{\alpha_{r}} / 2$ is the dimensionless critical normal crack opening displacement due to the adhesion energy of $\gamma_{s}$ (Zhang and Zhao 2012). This upward deformation of $\sqrt{\alpha_{r}} / 2$ at $\xi_{1}$ and $\xi_{2}$ is also demonstrated (by exaggeration) in Fig. 1a. The above two constraint conditions are obtained by minimizing the system total energy with respect to the suspension length. The detailed derivation of Eq. (11) is given by Zhang and Zhao (2011, 2012). When the axial load is nonzero, there is an additional energy due to stretching, which is $T \int_{-L_{1}}^{L_{2}} W_{x}^{2} d x / 2$. The stretching energy does not explicitly change the above constraint conditions. However, the presence of $T(\Delta)$ changes the solution forms as indicated above, the constraint conditions are thus implicitly changed.
Equations (9), (10) and (11) offer fourteen equations in total to solve the fourteen unknowns. Because of the unknown property of $\xi_{1}$ and $\xi_{2}$, solving these fourteen unknowns is a nonlinear problem, which requires NewtonRhapson method (Press et al. 1992). The above problem formulation is for the general case which includes asymmetric transverse loading scenario (Zhang and Zhao 2011, 2012). As shown later, a lot of iterations are needed to solve the inverse problem, which is very computational timeconsuming. The computation can be significantly reduced by using the symmetry property. The coordinate system is at the center of the beam, which is to say $l_{1}=l_{2}=l$. By using the symmetry property, the solution form of the zero axial load case of Eq. (6) now becomes the following

$w=\left\{\begin{array}{l}w_{1}=A_{1} \xi^{3}+B_{1} \xi^{2}+C_{1} \xi+D_{1} \\ w_{2}=B_{2} \cosh \xi \cos \xi+C_{2} \sinh \xi \sin \xi+h .\end{array}\right.$

Here we emphasize that stiction is defined as an attachment state under zero transverse load (de Boer and Michalske 1999; Legtenberg et al. 1994; Leseman et al. 2007; Mastrangelo and Hsu 1993b; Zhang and Zhao 2011, 2012). Therefore, $F=0$ and the terms associated with $F$ are gone. The $\cosh \xi \sin \xi$ and $\sinh \xi \cos \xi$ terms are tossed away because they are the symmetry-breaking odd functions. This technique of using the symmetry property to reduce computation was first done by Weitsman (1970) in the study of the tensionless contact of an infinitely long beam. Now there are only seven unknowns: $A_{1}, B_{1}, C_{1}, D_{1}, B_{2}, C_{2}$ and $\xi_{1}$.

The solution form of the tensile axial load case of Eq. (7) now becomes the following

$w=\left\{\begin{aligned} w_{1}= & A_{1} \cosh (\alpha \xi)+B_{1} \sinh (\alpha \xi)+C_{1} \xi+D_{1} \\ w_{2}= & B_{2} \cosh \left(f_{1} \xi\right) \cos \left(f_{2} \xi\right) \\ & +C_{2} \sinh \left(f_{1} \xi\right) \sin \left(f_{2} \xi\right)+h .\end{aligned}\right.$

Again, the $\cosh \left(f_{1} \xi\right) \sin \left(f_{2} \xi\right)$ and $\sinh \left(f_{1} \xi\right) \cos \left(f_{2} \xi\right)$ terms are tossed away because they are odd functions. Similarly, the solution form of the compressive axial load case of Eq. (8) now becomes the following

$w=\left\{\begin{aligned} w_{1}= & A_{1} \cos (\alpha \xi)+B_{1} \sin (\alpha \xi)+C_{1} \xi+D_{1} \\ w_{2}= & B_{2} \cosh \left(f_{1} \xi\right) \cos \left(f_{2} \xi\right) \\ & +C_{2} \sinh \left(f_{1} \xi\right) \sin \left(f_{2} \xi\right)+h\end{aligned}\right.$

Due to the symmetry of $\xi_{1}=-\xi_{2}$, only those matching conditions at $\xi=\xi_{1}$ are needed. Equation (9) is reduced to the following four:

$$
\begin{aligned}
& w_{1}\left(\xi_{1}\right)=w_{2}\left(\xi_{1}\right), \quad \frac{d w_{1}}{d \xi}\left(\xi_{1}\right)=\frac{d w_{2}}{d \xi}\left(\xi_{1}\right), \\
& \frac{d^{2} w_{1}}{d \xi^{2}}\left(\xi_{1}\right)=\frac{d^{2} w_{2}}{d \xi^{2}}\left(\xi_{1}\right), \quad \frac{d^{3} w_{1}}{d \xi^{3}}\left(\xi_{1}\right)=\frac{d^{3} w_{2}}{d \xi^{3}}\left(\xi_{1}\right)
\end{aligned}
$$


The boundary conditions now are only two:

$w_{1}(-l)=0, \quad \frac{d w_{1}}{d \xi}(-l)=0$.

The two constraint conditions are reduced to one:

$w_{2}\left(\xi_{1}\right)=h-\frac{\sqrt{\alpha_{r}}}{2}$.

Now Eqs. (15), (16) and (17) offer seven equations in total to solve the seven unknowns. For the Newton-Rhapson method (Press et al. 1992) to start, the initial guesses for the seven unknowns of $A_{1}, B_{1}, C_{1}, D_{1}, B_{2}, C_{2}$ and $\xi_{1}$ are needed. Except for the zero axial load case, both the tensile and compressive cases are sensitive to the initial guessed values. The general strategy here is to guess $\xi_{1}$ only and then find $A_{1}, B_{1}, C_{1}, D_{1}, B_{2}$ and $C_{2}$ by linear algebra from the guessed value of $\xi_{1}$.

\section{Results and discussion}

In this study, the dimensionless gap distance is fixed as $h=1$ for all the computation cases and $F=0$ because stiction is defined as an attachment state with zero transverse load. As mentioned above, the computation exhibits some sensitivity to the initial guessed equilibrium configuration. The presence of external load $F$ can relief such sensitivity. Wu et al. (2011) obtained the stiction equilibrium configuration by applying an external load and then gradually reducing it to zero. Let us first examine how our deflection shape differs from those of arc-shape and S-shape when the beam length is between the critical suspension lengths of arc-shape and S-shape. The previous description on the formation of arc- and S-shape is rather vague as follows: longer beam forms an S-shape and its unstuck/suspension length is appreciably shorter than the beam length; shorter beam forms an arc-shape and its unstuck/suspension length is approximately equal to the beam length (Rogers et al. 2002). In our study, the dimensionless suspension length is given as $s=l+\xi_{1}$. The dimensionless critical suspension length is $s_{c-c}=\sqrt[4]{36 h^{2} / \alpha_{r}}$ for S-shape and $s_{\text {arc }}=\frac{\sqrt{2}}{2} s_{c-c}$ for arc-shape as given in appendix. Now a natural question arises: what is the deflection shape of a beam when its length is between $s_{a r c}$ and $s_{c-c}$ ? This question seems a puzzle and concern to some researchers (Wu et al. 2011). For $h=1$ and $\alpha_{r}=1 \times 10^{-4}, s_{\text {arc }}=17.32$ and $s_{c-c}=24.5$. Figure 2 plots the deflection shapes of a beam with $l=20$, $\alpha_{r}=1 \times 10^{-4}$ and different $\Delta \mathrm{s}$ in comparison with arc and $\mathrm{S}$-shapes. There is little difference between the three deflection curves with three different $\Delta \mathrm{s}$ of $\Delta=0.02, \Delta=-0.02$ and $\Delta=0$. Their suspension lengths are all around $s=18.95$, which is also between $s_{\text {arc }}$ and $s_{c-c}$. When a compressive axial load is exerted, the caution for buckling

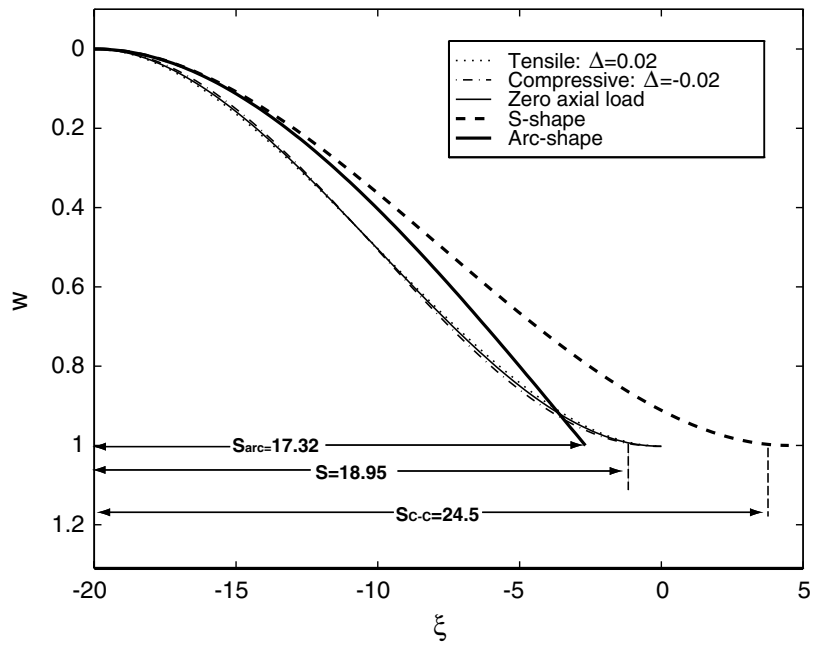

Fig. 2 Comparison of a "chunky" beam deflection shapes with arcshape and S-shape. The beam is with $\alpha_{r}=1 \times 10^{-4}$ and $l=20$, which is between $s_{a r c}$ and $s_{c-c}$. The three deformation shapes are the ones with $\Delta=2 \times 10^{-2}, \Delta=0$ and $\Delta=-2 \times 10^{-2}$, respectively

is needed. The governing equation of Eq. (2) is incapable of the post-buckling analysis. For a $\mathrm{C}-\mathrm{C}$ with the length of $2 L$, the critical buckling load is $T_{c}=-4 \pi^{2} E_{1} I /(2 L)^{2}$ (Chajes 1974), which corresponds to $\Delta_{c}=-\pi^{2} / l^{2}$ and $\Delta_{c} \approx-0.0247$ when $l=20$. Wu et al. (2011) speculated that the deflection of a beam with a length between $s_{a r c}$ and $s_{c-c}$ "may shift between the arc-shaped and S-shaped configuration, depending on the initial configurations." However, our computation shows that the deflection shapes look neither like arc-shape nor like S-shape, which is also experimentally observed (de Boer and Michalske 1999). de Boer and Michalske (1999) ascribed the mechanism to the compliance of support post. The arc-shape and S-shape as given in appendix actually prescribe the beam deflection shape by assuming the boundary conditions at the separation point. In contrast, our model does not prescribe the deflection shape, which is a function of $l, h, \Delta$ and $\alpha_{r}$. Physically, the difference between our model and arc/S-shape results from that the arc/S-shape method does not include the bending and contact energies in the stiction zone (Zhang and Zhao 2011, 2012). The arc/S-shape method is more or less inspired the classical study of mica splitting test by Obreimoff (1930), in which those energies inside the contact area are not included, either. However, there is one critical difference in the adhesion energy between the Obreimoff's mica splitting test and the microbeam stiction test. Obreimoff's (1930) splitting test is actually the cleavage of mica and the adhesion energy is "the extremely high value" of $20 \mathrm{Jm}^{-2}$, which is orders of magnitude larger than that of a stiction test. This large adhesion energy makes the deformation of a mica lamina inside contact area (unsplit part) extremely small compared with that of the split part. 


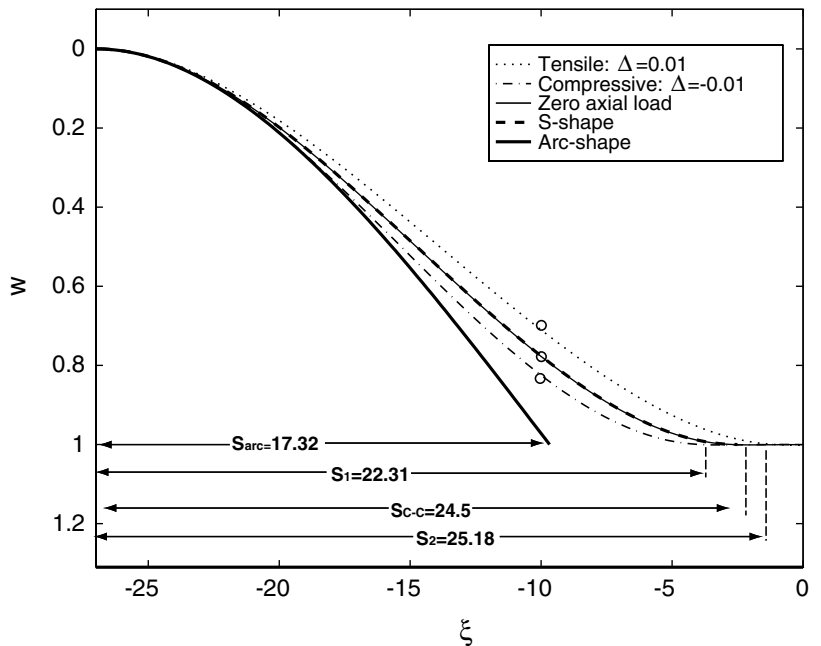

Fig. 3 Comparison of a "slender" beam deformation shapes with arc-shape and S-shape. The beam is with $\alpha_{r}=1 \times 10^{-4}$ and $l=27$, which is larger than $s_{c-c}$. The three deformation shapes are the ones with $\Delta=1 \times 10^{-2}, \Delta=0$ and $\Delta=-1 \times 10^{-2}$, respectively. The deflection curve of $\Delta=0$ overlaps with the S-shape. The circles mark the beam displacements at $\xi=-10$

Therefore, the bending and contact energies inside the contact zone can be ignored. Because of intrinsic surface roughness ( $\mathrm{Li}$ et al. 2010; Maboudian and Howe 1997; Tayebi and Polycarpou 2006; Wu et al. 2011; Zhang and Zhao 2011, 2012), nanoparticles formed during the fabrication process (DelRio et al. 2006), and presence of liquids (Legtenberg et al. 1994; Leseman et al. 2007; Tayebi and Polycarpou 2006; Wei and Zhao 2007), the nominal adhesion energy in a stiction test can vary with several orders of magnitudes. As listed in a reference (Zhang and Zhao 2012), the measured nominal adhesion energy of polysilicon ranges from $2.5 \times 10^{-4}$ to $0.27 \mathrm{Jm}^{-2}$. In comparison, the real adhesion energy of polysilicon computed by molecular dynamics is $2.54 \mathrm{Jm}^{-2}$ (Wu et al. 2011), which is still significantly smaller than that of the mica splitting test. Therefore, it is safe to keep both bending and contact energies for the stiction test case. Majidi and Adams (2009), Majidi and Fearing (2008) demonstrated that the bending energy of a film inside the contact zone is a vital factor impacting its adherence to a curvy body.

Figure 3 plots the deflection shapes of a slender beam with $l=27, \alpha_{r}=1 \times 10^{-4}$. Now the beam length is larger than $s_{c-c}$ and correspondingly the critical buckling load changes as $\Delta_{c} \approx-0.0135$. The three curves correspond to $\Delta=0.01, \Delta=-0.01$ and $\Delta=0$. $s_{\text {arc }}$ and $s_{c-c}$ are only dependent on $h$ and $\alpha_{r}$, which is to say that the variation of the beam length has no impact on them and they remain the same as $s_{c-c}=24.5$ and $s_{a r c}=17.32$. It is noticed in Fig. 3 that the deflection curve of $\Delta=0$ overlaps with $\mathrm{S}$-shape. Now the difference between the three deflections

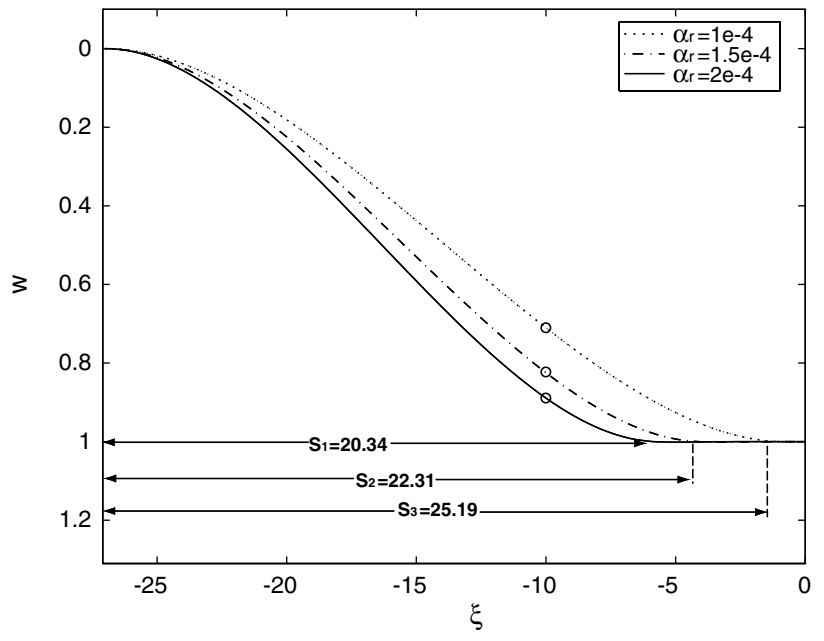

Fig. 4 Deflection shape variations as $\alpha_{r}$ changes. $l=27$ and $\Delta=1 \times 10^{-2}$ are fixed; $\alpha_{r}$ varies as $\alpha_{r}=1 \times 10^{-4}, \alpha_{r}=1.5 \times 10^{-4}$ and $\alpha_{r}=2 \times 10^{-4}$. The circles mark the beam displacements at $\xi=-10$

also becomes very significant: $s=22.31$ for $\Delta=-0.01$ ,$s=24.5$ for $\Delta=0$ and $s=25.18$ for $\Delta=0.01$. Stiction is the equilibrium due to the competition between the elastic restoring force which tries to pull the beam back to the horizontal position and the tensile force due to adhesion energy around the separation point which tries to keep the beam in contact (Zhang and Zhao 2011, 2012). The tensile axial load stiffens the beam, which increases the elastic restoring force and therefore, more beam portion peels off; the compressive axial load softens the beam, which decreases the elastic restoring and therefore, more beam portion adheres to the substrate. That the axial tensile/compressive load reduces/increases the attachment length has also been experimentally observed (Yee et al. 2002). As seen in Fig. 3, the suspension length monotonically increases as the axial load changes from compressive to tensile. Besides the suspension length variation, the solution forms of the beam deflection also vary as the axial load varies. As a result, the beam deflection shape difference stands out in the suspension zone. To evaluate this difference and also for the convenience of statement, we introduce a variable $r(\xi)=h-w_{1}(\xi)$. Physically, $r(\xi)$ is the beam rise above the substrate. In Fig. 3, at $\xi=-10$ , the three displacements of the beam under different axial loadings are marked with three circles. Of course, any place other than $\xi=-10$ can be selected to mark. It just seems that at $\xi=-10$, the difference maximizes. Clearly, $r(\xi)$ monotonically increases as the axial load changes from compressive to tensile: $r=0.175$ when $\Delta=-0.01$ $r=0.222$ when $\Delta=0$ and $r=0.282$ when $\Delta=0.01$. The physics behind this is that the beam deflection is different: different axial loadings give different suspension 


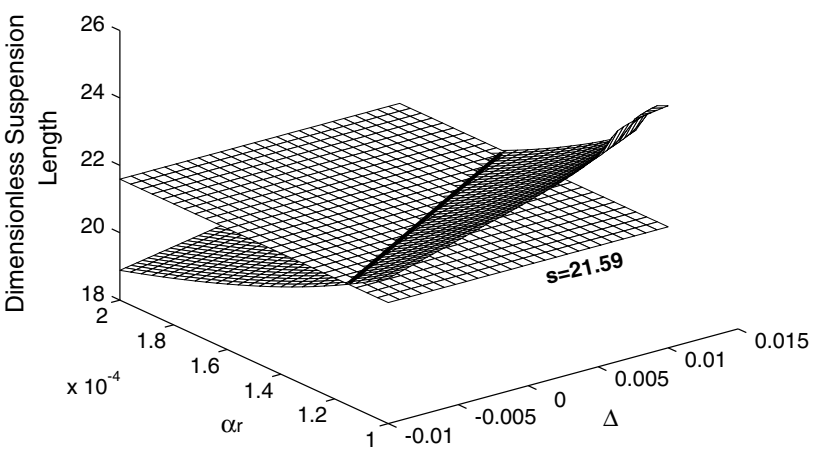

Fig. 5 The (dimensionless) suspension length variation as a function of $\alpha_{r}$ and $\Delta$. The level plane is the one with a constant suspension length of $s=21.59$. The two planes intersections are marked with a solid line

lengths and different mathematical solution forms. Figure 3 examines the effect of axial load on the beam with the fixed length and $\alpha_{r}$. In Fig. 4, we fix the beam length and axial load as $l=27$ and $\Delta=0.01$ to see how the beam deflection varies as a function of $\alpha_{r}$. As $\alpha_{r}$ varies as $\alpha_{r}=1 \times 10^{-4}, \alpha_{r}=1.5 \times 10^{-4}$ and $\alpha_{r}=2 \times 10^{-4}$, the suspension length monotonically decreases as $s=25.19$ ,$s=22.31$ and $s=20.34$. Larger $\alpha_{r}$ means larger adhesion energy influence, which thus leads to smaller suspension length. For arc and S-shapes which are the zero axial load case and have analytical expression for the suspension length, their suspension lengths have the relationship of $s \propto \alpha_{r}^{-1 / 4}$. The beam displacements at $\xi=-10$ are also marked with three circles. As $\alpha_{r}$ increases, $r$ decreases monotonically: $r=0.29$ when $\alpha_{r}=1 \times 10^{-4}, r=0.178$ when $\alpha_{r}=1.5 \times 10^{-4}$, and $r=0.111$ when $\alpha_{r}=2 \times 10^{-4}$ . Because the axial load is fixed as $\Delta=0.01$, the solution forms as given in Eq. (13) do not change. However, as $\alpha_{r}$ changes, the suspension length changes and as a result the coefficients associated with those solution forms change, which leads to the deflection shape difference.

As seen in Fig. 3, when $\alpha_{r}$ is fixed, both the beam suspension length and rise at a given point increase monotonically as $\Delta$ increases. In Fig. 4 , when $\Delta$ is fixed, both the beam suspension length and rise at a given point decrease monotonically as $\alpha_{r}$ increases. These monotonic increase and decrease properties are used to solve the inverse problem. To illustrate the inverse problem solving procedure, we give the example of $l=27, \alpha_{r}=1.5 \times 10^{-4}$ and $\Delta=5 \times 10^{-3}$, which gives the suspension length of $s=21.59$ and rise at $\xi=-10$ of $r=0.156$. In a stiction test, $\alpha_{r}$ and $\Delta$ are unknown; the beam dimensions, elastic properties, and beam deflections (i.e. $s$ and $r$ ) are measured. The main idea of solving this inverse problem is to search a wide range of $\alpha_{r}$ and $\Delta$ to see if there is a unique combination which satisfies $s=21.59$ and $r=0.156$.

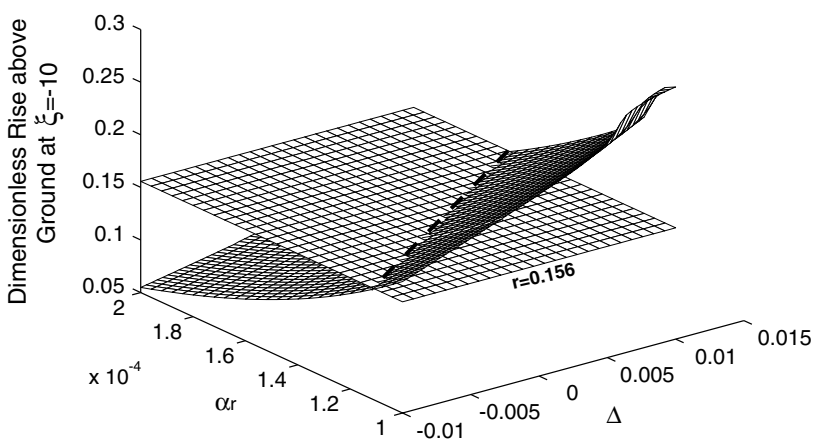

Fig. 6 The (dimensionless) rise variation as a function of $\alpha_{r}$ and $\Delta$. The level plane is the one with a constant rise of $r=0.156$. The two planes intersections are marked with a dashed line

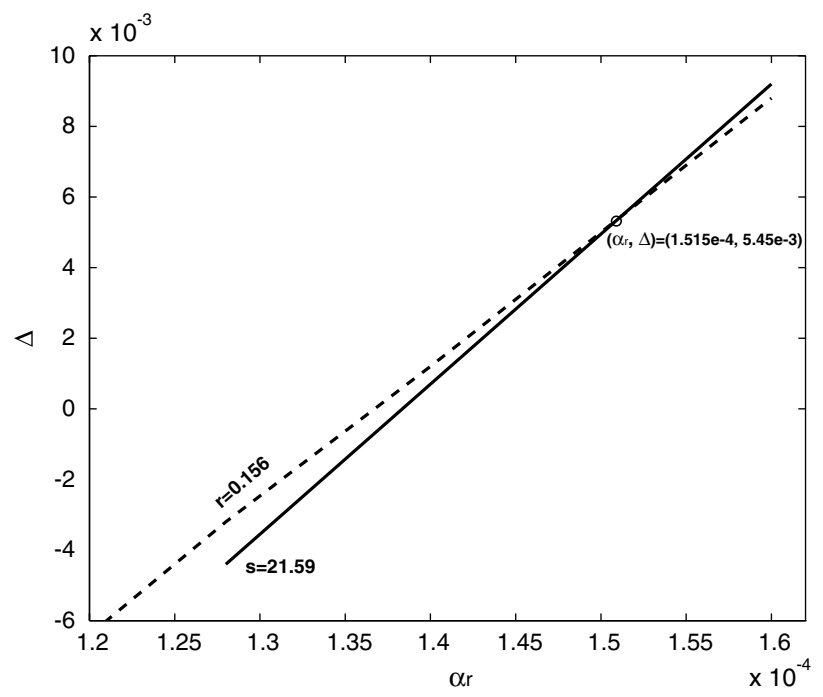

Fig. 7 The projections of the two intersection lines obtained in Figs. 5 and 6 into the $\alpha_{r}-\Delta$ plane. The intersection of the two lines is marked with a circle, which corresponds to $\left(\alpha_{r}, \Delta\right.$ )$=\left(1.515 \times 10^{-4}, 5.45 \times 10^{-3}\right)$

Figure 5 plots the suspension length variation as $\alpha_{r}$ varies from $1 \times 10^{-4}$ to $2 \times 10^{-4}$ and $\Delta$ varies from $-1 \times 10^{-2}$ to $1 \times 10^{-2}$. The variation step for $\alpha_{r}$ is $d \alpha_{r}=2 \times 10^{-6}$ and the variation step for $\Delta$ is $d \Delta=4 \times 10^{-4}$. The tilted plane indicates the suspension variation as a function of $\alpha_{r}$ and $\Delta$ . Again, as also seen in Figs. 3 and 4, for any given value of $\alpha_{r}$, the suspension length increases monotonically with $\Delta$. At the same time, for any given value of $\Delta$, the suspension length decreases monotonically with $\alpha_{r}$. The level plane is the one with the fixed suspension length of $s=21.59$. The intersection of these two planes are marked by a solid line. Physically, this line indicates that there are infinite combinations of $\alpha_{r}$ and $\Delta$ which can result in the same suspension length of $s=21.59$ for a beam with $l=27$. In other words, measuring the suspension length alone cannot uniquely 
determine $\alpha_{r}$ and $\Delta$. Analogously, Knapp and de Boer (2002) showed that different combinations of transverse external load and adhesion energy can result in the same suspension length. Figure 6 plots the beam rise variation as $\alpha_{r}$ varies from $1 . \times 10^{-4}$ to $2 \times 10^{-4}$ and $\Delta$ varies from $-1 \times 10^{-2}$ to $1 \times 10^{-2}$. As also seen in Figs. 3 and 4 , the same monotonic increase and decrease patterns of $r$ occur. The level plane is the one with the fixed beam rise of $r=0.156$ (at $\xi=-10)$. The intersection of these two planes are marked by a dashed line. Again, this dashed line indicates that for the same beam rise, there are infinite combinations of $\alpha_{r}$ and $\Delta$. Measuring the beam rise at a given point alone cannot uniquely determine $\alpha_{r}$ and $\Delta$, either. When the two intersecting lines obtained in Figs. 5 and 6 are projected into the $\alpha_{r}-\Delta$ plane, the two lines intersect as shown in Fig. 7. The intersection point is marked with a circle, which corresponds to $\left(\alpha_{r}, \Delta\right)=\left(1.515 \times 10^{-4}, 5.45 \times 10^{-3}\right)$. Compared with $\left(\alpha_{r}, \Delta\right)=\left(1.5 \times 10^{-4}, 5 \times 10^{-3}\right)$ which results in $s=21.59$ and $r=0.156$, the error of $\alpha_{r}$ is $1 \%$ and the error of $\Delta$ is $9 \%$. The accuracy of $\alpha_{r}$ and $\Delta$ can be further improved by taking smaller steps with the trade-off of more computation efforts.

Here the inverse problem is solved by the use of suspension length and rise at a given point. As the deflection of a stuck beam can be accurately measured (de Boer and Michalske 1999; Jensen et al. 2001; Mastrangelo and Hsu 1993b), a direct deflection shape comparison between the experimental measured one and those given in Eqs. (6), (7) and (8) by varying axial load and adhesion energy ( $\Delta$ and $\alpha_{r}$ ) is also another method of solving the inverse problem.

\section{Conclusion}

The residual stress effect is modeled as an axial load, which can either stiffen or soften the beam. Mathematically, the presence of axial load changes the solution forms of the beam; the effect of adhesion energy is embodied in the constraint condition. For a microbeam with the given dimensions and elastic properties, the axial load and adhesion energy uniquely determine its stiction shape. In this study, the beam stiction shape is characterized by the suspension length and rise at a given point. The axial load and adhesion energy play different roles in determining the beam stiction shape, which results in the monotonic increase and decrease patterns of the suspension length and rise. These patterns are used in this study to solve the inverse problem. For a given value of either suspension length or rise, there are infinite combinations of axial load and adhesion energy. However, if both values are given, there is only one combination. This study presents an effective method to solve this type of inverse problem though it requires relatively large amounts of computation for large amounts of the combinations of axial load and adhesion energy. Because the deflection of a chunky beam is much less sensitive to the axial load variation, the method is suitable for the slender beam structure.

Acknowledgments The research has been supported by the National Natural Science Foundation of China (NSFC Nos. 11023001 and 11372321).

\section{Appendix: arc-shaped and S-shaped deflections}

Figure $1 \mathrm{~b}$ shows the arc-shape and S-shaped deflections and the coordinate system. When a beam is under stiction with no transverse and axial loads, the following governing equation holds:

$w_{\xi \xi \xi \xi}=0$.

The solution to the above equation is

$w(\xi)=A \xi^{3}+B \xi^{2}+C \xi+D$.

$A, B, C$ and $D$ are the four unknown constants to be determined by the boundary conditions.

For an arc-shaped beam, the following boundary conditions hold:

$w(0)=0, \quad w_{\xi}(0)=0, \quad w\left(s_{\text {arc }}\right)=h, \quad w_{\xi \xi}\left(s_{\text {arc }}\right)=(20)$ $w(\xi)$ of arc-shaped beam is then solved as follows

$w(\xi)=-\frac{h}{2 s_{\text {arc }}^{3}} \xi^{3}+\frac{3 h}{2 s_{\text {arc }}^{2}} \xi^{2}$.

The (dimensional) suspension length of arc-shaped beam is given as follows (Mastrangelo and Hsu 1993b; Yang 2004)

$S_{\text {arc }}^{4}=\frac{3}{8} \frac{E_{1} H^{2} T^{3}}{\gamma_{s}}=\frac{9 E_{1} I H^{2}}{4 B \gamma_{s}}$.

According to the nondimensionalization scheme of Eq. (4), we have $s_{\text {arc }}=\beta S_{\text {arc }}=\sqrt[4]{9 h^{2} / \alpha}$.

For an S-shaped beam, the following boundary conditions hold:

$w(0)=0, \quad w_{\xi}(0)=0, \quad w\left(s_{c-c}\right)=h, \quad w_{\xi}\left(s_{c-c}\right)=(23)$ $w(\xi)$ of S-shaped beam is solved as follows

$w(\xi)=-\frac{2 h}{s_{c-c}^{3}} \xi^{3}+\frac{3 h}{s_{c-c}^{2}} \xi^{2}$.

The (dimensional) suspension length of S-shaped beam is given by Yang (2004) as follows

$S_{c-c}^{4}=\frac{3}{2} \frac{E_{1} H^{2} T^{3}}{\gamma_{s}}=\frac{9 E_{1} I H^{2}}{B \gamma_{s}}$.

The dimensionless $s_{c-c}=\beta S_{c-c}=\sqrt[4]{36 h^{2} / \alpha_{r}}$ and $s_{\text {arc }}=\frac{\sqrt{2}}{2} s_{c-c}$. Equations (21) and (24) are also presented 
by de Boer et al. (1999). As the two coordinate systems in Fig. 1a and b are different, Eqs. (21) and (24) are the following forms in the coordinate system of Fig. 1a

$$
\begin{aligned}
& w(\xi)=-\frac{h}{2 s_{\text {arc }}^{3}}\left(\xi+l_{1}\right)^{3}+\frac{3 h}{2 s_{\text {arc }}^{2}}\left(\xi+l_{1}\right)^{2} \quad(\text { arc - shape }), \\
& w(\xi)=-\frac{2 h}{s_{c-c}^{3}}\left(\xi+l_{1}\right)^{3}+\frac{3 h}{s_{c-c}^{2}}\left(\xi+l_{1}\right)^{2} \quad(S-\text { shape }) .
\end{aligned}
$$

\section{References}

Biot MA (1937) Bending of an infinite beam on an elastic foundation. ASME J Appl Mech 4:1-7

Castillo J, Barber JS (1997) Lateral contact of slender prismatic bodies. Proc R Soc Lond A 453:2397-2412

Chajes A (1974) Principles of structural stability theory. PrenticeHall, New Jersey

Chen RS, Baughn TV, Yao Z, Goldsmit CL (2002) A new in-situ residual stress measurement method for a MEMS thin fixed-fixed structure. J Microelectromech Syst 11:309-316

de Boer MP, Michalske TA (1999) Accurate method for determining adhesion of cantilever beams. J Appl Phys 86:817-827

de Boer MP, Knapp JA, Mayer TM, Michalske TA (1999) The role of interfacial properties on MEMS performance and reliability. Proc SPIE 3825:2-15

DelRio F, de Boer MP, Knapps JA, Reedy E Jr, Clews P, Dunn M (2005) The role of van der Waals forces in adhesion of micomachined surfaces. Nature Mat 4:629-634

DelRio F, Dunn ML, Boyce BL, Corwin AD, de Boer MP (2006) The effect of nanoparticles on rough surface adhesion. J Appl Phys 99:104304

Ghatak A, Mahadevan L, Chung JY, Chaudhury MK, Shenoy V (2004) Peeling from a biomimetically patterned thin elastic film. Proc R Soc Lond A 460:2725-2735

Ghatak A, Mahadevan L, Chaudhury MK (2005) Measuring the work of adhesion between a soft confined film and a flexible plate. Langmuir 21:1277-1281

Jensen BD, de Boer MP, Masters ND, Bitsie FD, LaVan A (2001) Interferometry of actuated microcantilevers to determine material properties and test structure nonidealities in MEMS. J Microelectromech Syst 10:336-346

Jiang H, Sun Y, Rogers JA, Huang Y (2008) Post-buckling analysis for the precisely controlled buckling of thin film encapsulated by elastomeric substrate. Int J Solids Struct 45:2014-2023

Jones EE, Begley MR, Murphy KD (2003) Adhesion of micro-cantilever subjected to mechanical point loading: modeling and experiments. J Mech Phys Solids 51:1601-1622

Kerr AD (1976) On the derivation of well posed boundary value problems in structural mechanics. Int J Solids Struct 12:1-11

Knapp JA, de Boer MP (2002) Mechanics of microcantilever beams subjected to combined electrostatic and adhesive forces. J Microelectromech Syst 11:754-764

Komaragiri U, Begley MR, Simmonds JG (2005) The mechanical response of freestanding circular elastic films under point and pressure loads. ASME J Appl Mech 72:203-212

Legtenberg R, Tilmans T, Elders J, Elwenspoek M (1994) Stiction of surface micromachined structures after rinsing and drying: model and investigation of adhesion mechanisms. Sens Actuators A 43:230-238

Leseman ZC, Carlson SP, Mackin TJ (2007) Experimental measurements of the strain energy release rate for stiction-failed microcantilevers using a single-cantilever beam peel test. J Microelectromech Syst 16:38-43

Li GH, Laboriante I, Liu F, Shavezipur M, Bush B, Carraro C, Maboudian R (2010) Measurement of adhesion forces between polycrystalline silicon surfaces via a MEMS double-clamped beam test structure. J Micromech Microeng 20:095015

Lin L, Adams GG (1987) Beam on tensionless elastic foundation. J Eng Mech 113:542-553

Liu JL (2010) Theoretical analysis on capillary adhesion of microsized plates with a substrate. Acta Mech Sin 26:217-223

Loh OY, Espinosa HD (2012) Nanoelectromechanical contact switches. Nature Nanotech 7:283-295

Maboudian R, Howe RT (1997) Critical review: adhesion in surface micromechanical structures. J Vac Sci Technol B 15:1-20

Majidi CS, Adams GG (2009) A simplified formulation of adhesion problems with elastic plates. Proc R Soc Lond A 465:2217-2230

Majidi CS, Fearing RS (2008) Adhesion of an elastic plate to a sphere. Proc R Soc Lond A 464:1309-1317

Majidi CS, Groff RE, Fearing RS (2005) Attachment of fiber array adhesive through side contact. J Appl Phys 98:103521

Mastrangelo CH, Hsu CH (1993a) Mechanical stability and adhesion of microstructures under capillary forces-part I: basic theory. J Microelectromech Syst 2:33-43

Mastrangelo CH, Hsu CH (1993b) Mechanical stability and adhesion of microstructures under capillary forces-part II: experiments. J Microelectromech Syst 2:44-55

Obreimoff JW (1930) The splitting strength of mica. Proc R Soc Lond A 127:290-297

Press WH, Teukolsky SA, Vetterling WT, Flannery BP (1992) Numerical recipes in fortran, 2nd edn, chap.9. Cambridge University Press, Cambridge

Rogers JW, Mackin TJ, Phinney LM (2002) A thermomechanical model for adhesion reduction of MEMS cantilevers. J Microelectromech Syst 11:512-520

Tayebi N, Polycarpou AA (2006) Adhesion and contact modeling and experiments in microelectromechanical systems including roughness effects. Microsyst Technol 12:854-869

van Spengen WM, Puers R, Mertents R, De Wolf I (2004) Characterization and failure analysis of MEMS: high resolution optical investigation of small out-of-plane movements and fast vibrations. Microsyst Technol 10:89-96

Vlassak JJ, Nix WD (1992) A new bulge test for the determination of Young's modulus and Poisson's ratio of thin film. J Mater Res 7:3242-3249

Wei Z, Zhao YP (2007) Growth of liquid bridge in AFM. J Phys D Appl Phys 40:4368-4375

Weitsman Y (1970) On foundations that reacts in compression only. ASME J Appl Mech 37:1019-1030

Wong MF, Duan G, Wan KT (2007) Adhesion-delamination mechanics of a prestressed rectangular film adhered onto a rigid substrate. J Appl Phys 101:024903

Wu L, Noels L, Rochus V, Pustan M, Golinval JC (2011) A micromacroapproach to predict stiction due to surface contact in microelectromechanical systems. J Microelectromech Syst 20:976-990

Wu L, Golinval JC, Noels L (2013) A micro-model for elasto-plastic adhesive-contact in micro-switches: application to cyclic loading. Tribol Int 57:137-146

Yang F (2004) Contact deformation of a micromechanical structure. J Micromech Microeng 14:263-268

Yang F (2006) Electromechanical contact of microelectromechanical structure. J Appl Phys 100:124511

Yee Y, Park M, Chun K (2002) A sticking model of suspended polysilicon microstructure including residual stress gradient and postrelease temperature. J Microelectromech Syst 11:754-764

Zhang Y (2008) Tensionless contact of a finite beam resting on Reissner foundation. Int J Mech Sci 50:1035-1041 
Zhang Y, Murphy KD (2004) Response of a finite beam in contact with a tensionless foundation under symmetric and asymmetric loading. Int J Solids Struct 41:6745-6758

Zhang Y, Zhao YP (2004) Static study of cantilever beam stiction and electrostatic force influence. Acta Mech Solida Sin 17:104-112

Zhang Y, Zhao YP (2006) An effective method of determining the residual stress gradients in a micro-cantilever. Microsyst Technol $12: 357-364$
Zhang Y, Zhao YP (2011) A precise model for the shape of an adhered microcantilever. Sens. Actuators A 171:381-390

Zhang Y, Zhao YP (2012) Flexural contact in MEMS stiction. Int J Solids Struct 49:2203-2214

Zhao YP, Wang LS, Yu TX (2003) Mechanics of adhesion in MEMSa review. J Adhes Sci Tech 17:519-546 